\title{
Running CP-violating Phases of Majorana Neutrinos
}

\section{Zhi-zhong Xing ${ }^{* \dagger}$}

Institute of High Energy Physics, Chinese Academy of Sciences, Beijing 100049, China

E-mail: xingzz@mail.ihep.ac.cn

\begin{abstract}
Three CP-violating phases of the $3 \times 3$ lepton flavor mixing matrix $V$, denoted as $\delta, \rho$ and $\sigma$ in a useful phase convention, are entangled with one another in the one-loop renormalization-group equations. We show that $\delta=90^{\circ}$ at the electroweak scale $\left(\Lambda_{\mathrm{EW}} \sim 10^{2} \mathrm{GeV}\right)$ can be radiatively generated from $\delta=0^{\circ}$ at the seesaw scale $\left(\Lambda_{\mathrm{SS}} \sim 10^{14} \mathrm{GeV}\right)$ in the minimal supersymmetric standard model, provided three neutrino masses are nearly degenerate. As for the Majorana phases $\rho$ and $\sigma$, it is also possible to radiatively generate $\rho=90^{\circ}$ or $\sigma=90^{\circ}$ at $\Lambda_{\mathrm{EW}}$ from $\rho=0^{\circ}$ or $\sigma=0^{\circ}$ at $\Lambda_{\mathrm{SS}}$. This observation opens a new and interesting window for model building, in order to understand possible connection between the phenomena of CP violation at low- and high-energy scales.
\end{abstract}

International Europhysics Conference on High Energy Physics

July 21st - 27th 2005

Lisboa, Portugal

\footnotetext{
* Speaker.

$\dagger$ In collaboration with S. Luo and J.W. Mei (hep-ph/0507065), and supported in part by the National Natural Science Foundation of China
} 
Recent solar and atmospheric neutrino oscillation experiments have provided us with very robust evidence that neutrinos are massive and lepton flavors are mixed. A useful parametrization of the $3 \times 3$ lepton flavor mixing matrix $V$ reads [1]:

$$
V=\left(\begin{array}{ccc}
c_{12} c_{13} & s_{12} c_{13} & s_{13} \\
-c_{12} s_{23} s_{13}-s_{12} c_{23} e^{-i \delta} & -s_{12} s_{23} s_{13}+c_{12} c_{23} e^{-i \delta} & s_{23} c_{13} \\
-c_{12} c_{23} s_{13}+s_{12} s_{23} e^{-i \delta} & -s_{12} c_{23} s_{13}-c_{12} s_{23} e^{-i \delta} & c_{23} c_{13}
\end{array}\right)\left(\begin{array}{ccc}
e^{i \rho} & 0 & 0 \\
0 & e^{i \sigma} & 0 \\
0 & 0 & 1
\end{array}\right),
$$

where $c_{i j} \equiv \cos \theta_{i j}$ and $s_{i j} \equiv \sin \theta_{i j}$. Current data yield $\theta_{12} \approx 33^{\circ}, \theta_{23} \approx 45^{\circ}$ and $\theta_{13}<10^{\circ}$ [2], but $\delta, \rho$ and $\sigma$ remain entirely unrestricted. Many new neutrino experiments are underway, not only to measure $\theta_{13}$ and $\delta$, but also to constrain the Majorana phases $\rho$ and $\sigma$.

While neutrino masses and lepton flavor mixing parameters can be measured at low-energy scales, their origin is most likely to depend on some unspecified interactions at a superhigh energy scale. For instance, the existence of very heavy right-handed neutrinos and lepton number violation may naturally explain the smallness of left-handed neutrino masses via the famous seesaw mechanism at the scale $\Lambda_{\mathrm{SS}} \sim 10^{14} \mathrm{GeV}$. Below this seesaw scale, the effective Lagrangian for lepton Yukawa interactions can be written as $-\mathscr{L}=\overline{E_{L}} H_{1} Y_{l} l_{R}-\overline{E_{L}} H_{2}(\kappa / 2) H_{2}^{c \dagger} E_{L}^{c}+$ h.c. in the minimal supersymmetric standard model (MSSM). After spontaneous gauge symmetry breaking at the electroweak scale $\Lambda_{\mathrm{EW}} \sim 10^{2} \mathrm{GeV}$, we arrive at the charged lepton mass matrix $M_{l}=\mathbf{v} Y_{l} \cos \beta$ and the effective neutrino mass matrix $M_{v}=\mathbf{v}^{2} \kappa \sin ^{2} \beta$. The lepton flavor mixing matrix $V$ arises from the mismatch between the diagonalizations of $Y_{l}$ (or $M_{l}$ ) and $\kappa$ (or $M_{v}$ ). In the flavor basis where $Y_{l}$ is diagonal, $V$ directly links the neutrino mass and flavor eigenstates. The physical parameters at $\Lambda_{\mathrm{SS}}$ and $\Lambda_{\mathrm{EW}}$ are related by the renormalization group equations (RGEs). It has been shown that the RGE evolution between these two scales may have significant effects on the mixing angle $\theta_{12}$ and the phase parameters $\delta, \rho$ and $\sigma$, and a CP-violating phase can even be radiatively generated [3].

The reason for the radiative generation of a CP-violating phase is simply that three phases of $V$ are entangled with one another in the RGEs. Hence a non-zero value of $\delta$ can be generated at $\Lambda_{\mathrm{EW}}$ from $\delta=0$ at $\Lambda_{\mathrm{SS}}$, provided the initial values of $\rho$ and $\sigma$ are not vanishing. This observation opens a new and interesting window to understand possible connection between the phenomena of $\mathrm{CP}$ violation at low- and high-energy scales; e.g., the phase parameter governing the strength of $\mathrm{CP}$ violation in neutrino oscillations could be radiatively generated from those $\mathrm{CP}$-violating phases which control the leptogenesis of right-handed neutrinos at $\Lambda_{\mathrm{SS}}$. The purpose of this talk is just to illustrate the maximal radiative generation of $\delta, \rho$ and $\sigma$ in the RGE evolution from $\Lambda_{\mathrm{SS}}$ to $\Lambda_{\mathrm{EW}}$.

Below $\Lambda_{\mathrm{SS}}$, the one-loop RGE of $\kappa$ in the MSSM can be found in Ref. [3]. In the flavor basis where $Y_{l}$ is diagonal, we have $\kappa=V \bar{\kappa} V^{T}$ with $\bar{\kappa}=\operatorname{Diag}\left\{\kappa_{1}, \kappa_{2}, \kappa_{3}\right\}$. The neutrino masses at $\Lambda_{\mathrm{EW}}$ read as $m_{i}=\mathbf{v}^{2} \kappa_{i} \sin ^{2} \beta$ (for $i=1,2,3$ ). It is then possible to derive the RGEs for $\left(\kappa_{1}, \kappa_{2}, \kappa_{3}\right)$, $\left(\theta_{12}, \theta_{23}, \theta_{13}\right)$ and $(\delta, \rho, \sigma)[4]$. For simplicity, here we only quote the approximate RGEs of $\delta, \rho$ and $\sigma$ in the working assumption that three neutrino masses are nearly degenerate [4]:

$$
\begin{aligned}
\frac{\mathrm{d} \delta}{\mathrm{d} t} & \approx \frac{y_{\tau}^{2}}{16 \pi^{2}}\left[\frac{c_{(\rho-\sigma)}^{c_{(\rho-\sigma)}}}{\zeta_{12}} s_{23}^{2}+\left(\frac{c_{(\delta-\rho)} s_{\rho}}{\zeta_{13}}-\frac{c_{(\delta-\sigma)} s_{\sigma}}{\zeta_{23}}\right) \frac{c_{12} s_{12} c_{23} s_{23}}{s_{13}}\right], \\
\frac{\mathrm{d} \rho}{\mathrm{d} t} & \approx \frac{y_{\tau}^{2}}{16 \pi^{2}}\left[\frac{c_{(\rho-\sigma)} s_{(\rho-\sigma)}}{\zeta_{12}} s_{12}^{2} s_{23}^{2}+\left(\frac{c_{(\delta-\rho) s}}{\zeta_{13}}-\frac{c_{(\delta-\sigma)} s_{\sigma}}{\zeta_{23}}\right) \frac{c_{12} s_{12} c_{23} s_{23}}{s_{13}}\right], \\
\frac{\mathrm{d} \sigma}{\mathrm{d} t} & \approx \frac{y_{\tau}^{2}}{16 \pi^{2}}\left[\frac{c_{(\rho-\sigma)} c_{(\rho-\sigma)}}{\zeta_{12}} c_{12}^{2} s_{23}^{2}+\left(\frac{c_{(\delta-\rho) s \rho}}{\zeta_{13}}-\frac{c_{(\delta-\sigma)} s_{\sigma}}{\zeta_{23}}\right) \frac{c_{12} s_{12} c_{23} s_{23}}{s_{13}}\right],
\end{aligned}
$$


Table 1: Radiative generation of $\delta=90^{\circ}$ in the MSSM with $\tan \beta=10$.

\begin{tabular}{c|l|ccc}
\hline \hline Parameter & Input $\left(\Lambda_{\mathrm{SS}}\right)$ & \multicolumn{3}{|c}{ Output $\left(\Lambda_{\mathrm{EW}}\right)$} \\
& & $\theta_{13}=1^{\circ}$ & $\theta_{13}=3^{\circ}$ & $\theta_{13}=5^{\circ}$ \\
\hline$m_{1}(\mathrm{eV})$ & 0.241 & 0.20 & 0.20 & 0.20 \\
$\Delta m_{21}^{2}\left(10^{-5} \mathrm{eV}^{2}\right)$ & 20.4 & 7.79 & 7.17 & 6.56 \\
$\Delta m_{31}^{2}\left(10^{-3} \mathrm{eV}^{2}\right)$ & 3.32 & 3.20 & 2.20 & 2.20 \\
\hline$\theta_{12}$ & $24.1^{\circ}$ & $45.0^{\circ}$ & $33.0^{\circ}$ & $33.1^{\circ}$ \\
$\theta_{23}$ & $43.9^{\circ}$ & $0.65^{\circ}$ & $2.46^{\circ}$ & $45.0^{\circ}$ \\
$\theta_{13}$ & $1^{\circ} / 3^{\circ} / 5^{\circ}$ & $90.0^{\circ}$ & $41.8^{\circ}$ & $35.8^{\circ}$ \\
$\delta$ & $0^{\circ}$ & $72.2^{\circ}$ & $23.8^{\circ}$ & $17.6^{\circ}$ \\
$\rho$ & $4.0^{\circ}$ & $26.3^{\circ}$ & $-22.0^{\circ}$ & $-28.1^{\circ}$ \\
$\sigma$ & $-57.5^{\circ}$ & \multicolumn{4}{c}{} \\
\hline \hline
\end{tabular}

where $\zeta_{i j} \equiv\left(\kappa_{i}-\kappa_{j}\right) /\left(\kappa_{i}+\kappa_{j}\right), c_{(\rho-\sigma)} \equiv \cos (\rho-\sigma), s_{(\rho-\sigma)} \equiv \sin (\rho-\sigma)$, etc. One can see that the one-loop RGE running behaviors of $\delta, \rho$ and $\sigma$ are very similar to one another.

I proceed to show a few typical numerical examples. The eigenvalues of $Y_{l}$ at $\Lambda_{\mathrm{SS}}$ are chosen in such a way that they can correctly run to their low-energy values. We assume the masses of three light neutrinos to be nearly degenerate and $m_{1} \sim 0.2 \mathrm{eV}$, so as to make the RGE running effects of relevant physical quantities significant enough. The initial values of $\kappa_{i}$ can be adjusted via $\kappa_{i}=m_{i} /\left(\mathbf{v}^{2} \sin ^{2} \beta\right)$ together with a typical input $\tan \beta=10$, such that the resultant neutrino masssquared differences $\Delta m_{21}^{2} \equiv m_{2}^{2}-m_{1}^{2}$ and $\Delta m_{31}^{2} \equiv m_{3}^{2}-m_{1}^{2}$ at $\Lambda_{\mathrm{EW}}$ are consistent with the solar and atmospheric neutrino oscillation data. We follow a similar strategy to choose the initial values of three mixing angles $\theta_{12}, \theta_{23}$ and $\theta_{13}$, in order to reproduce their low-energy values determined or constrained by current experimental data [2]. In view of the upper bound $\theta_{13}<10^{\circ}$, we shall typically take $\theta_{13}=1^{\circ}, 3^{\circ}$ and $5^{\circ}$ in our numerical calculations. We allow one of three CP-violating phases to vanish at $\Lambda_{\mathrm{SS}}$ and examine whether it can run to $90^{\circ}$ at $\Lambda_{\mathrm{EW}}$ by choosing the initial values of the other two phase parameters properly.

(A) Radiative generation of $\delta=90^{\circ}$. The input and output values of relevant parameters are listed in Table 1 . We see that $\delta=90^{\circ}$ at $\Lambda_{\mathrm{EW}}$ can be radiatively generated from $\delta=0^{\circ}$ at $\Lambda_{\mathrm{SS}}$, if $\theta_{13}=1^{\circ}, \rho=4.0^{\circ}$ and $\sigma=-57.5^{\circ}$ are input. Changing the initial value of $\theta_{13}$ to $3^{\circ}$ or $5^{\circ}$ but fixing the input values of the other quantities, we find that only $\delta=41.8^{\circ}$ or $\delta=35.8^{\circ}$ can be obtained at $\Lambda_{\mathrm{EW}}$. While the results of $m_{1}, \Delta m_{31}^{2}, \theta_{12}$ and $\theta_{23}$ at $\Lambda_{\mathrm{EW}}$ are rather stable against the change of $\theta_{13}$ from $1^{\circ}$ to $5^{\circ}$ at $\Lambda_{\mathrm{SS}}$, the result of $\Delta m_{21}^{2}$ becomes smaller and less favored.

Next let us examine whether the radiative generation of $\delta=90^{\circ}$ at $\Lambda_{\mathrm{EW}}$ can be achieved from other initial values of $\rho$ and $\sigma$, when $\theta_{13}=1^{\circ}$ holds and $m_{1}, \Delta m_{21}^{2}, \Delta m_{31}^{2}, \theta_{12}$ and $\theta_{23}$ take the same input values as before at $\Lambda_{\mathrm{SS}}$ (see Table 1). We find out two new numerical examples with $(\rho, \sigma)=\left(0^{\circ},-59.9^{\circ}\right)$ and $\left(10^{\circ},-57^{\circ}\right)$, respectively. Note that both $\delta$ and $\rho$ (or $\left.\sigma\right)$ can be radiatively generated from $\sigma \neq 0^{\circ}$ (or $\rho \neq 0^{\circ}$ ) at the seesaw scale. The possibility to simultaneously generate $\rho$ and $\sigma$ from $\delta \neq 0^{\circ}$ at $\Lambda_{\mathrm{SS}}$ via the RGE evolution is in general expected to be strongly suppressed, because the leading terms of $\mathrm{d} \rho / \mathrm{d} t$ and $\mathrm{d} \sigma / \mathrm{d} t$ in Eq. (2) vanish for $\rho=\sigma=0^{\circ}$ at 
Table 2: Radiative generation of $\rho=90^{\circ}$ in the MSSM with $\tan \beta=10$.

\begin{tabular}{c|l|ccc}
\hline \hline Parameter & Input $\left(\Lambda_{\mathrm{SS}}\right)$ & \multicolumn{3}{|c}{ Output $\left(\Lambda_{\mathrm{EW}}\right)$} \\
& & $\theta_{13}=1^{\circ}$ & $\theta_{13}=3^{\circ}$ & $\theta_{13}=5^{\circ}$ \\
\hline$m_{1}(\mathrm{eV})$ & 0.241 & 0.20 & 0.20 & 0.20 \\
$\Delta m_{21}^{2}\left(10^{-5} \mathrm{eV}^{2}\right)$ & 20.4 & 8.54 & 7.90 & 7.27 \\
$\Delta m_{31}^{2}\left(10^{-3} \mathrm{eV}^{2}\right)$ & 3.32 & 2.21 & 2.20 & 2.20 \\
\hline$\theta_{12}$ & $27.6^{\circ}$ & $33.1^{\circ}$ & $33.2^{\circ}$ & $33.3^{\circ}$ \\
$\theta_{23}$ & $43.9^{\circ}$ & $44.8^{\circ}$ & $44.8^{\circ}$ & $44.8^{\circ}$ \\
$\theta_{13}$ & $1^{\circ} / 3^{\circ} / 5^{\circ}$ & $0.43^{\circ}$ & $2.17^{\circ}$ & $4.24^{\circ}$ \\
\hline$\delta$ & $0^{\circ}$ & $107.6^{\circ}$ & $35.4^{\circ}$ & $30.2^{\circ}$ \\
$\rho$ & $0^{\circ}$ & $90.2^{\circ}$ & $17.6^{\circ}$ & $12.1^{\circ}$ \\
$\sigma$ & $-67.7^{\circ}$ & $34.1^{\circ}$ & $-38.3^{\circ}$ & $-44.6^{\circ}$ \\
\hline \hline
\end{tabular}

$\Lambda_{\mathrm{SS}}$. Only in the $\theta_{13} \rightarrow 0$ limit, the running effects of three CP-violating phases could become significant. Our results hint at the existence of strong parameter degeneracy in obtaining $\delta=90^{\circ}$ at $\Lambda_{\mathrm{EW}}$ from $\delta=0^{\circ}$ at $\Lambda_{\mathrm{SS}}$. To resolve this problem is certainly a big challenge in model building, unless two Majorana CP-violating phases could separately be measured at low energies.

(B) Radiative generation of $\rho=90^{\circ}$ or $\sigma=90^{\circ}$. Now we take a look at the one-loop RGE evolution of $\rho$. The inputs and outputs of relevant parameters are listed in Table 2. One can see that there is no difficulty to radiatively generate $\rho=90^{\circ}$ at $\Lambda_{\mathrm{EW}}$ from $\rho=0^{\circ}$ at $\Lambda_{\mathrm{SS}}$, provided $\theta_{13}=1^{\circ}$, $\delta=0^{\circ}$ and $\sigma=-67.7^{\circ}$ are input. Allowing the initial value of $\theta_{13}$ to change to $3^{\circ}$ or $5^{\circ}$ but fixing the input values of the other quantities, we find that only $\rho=17.6^{\circ}$ or $\rho=12.1^{\circ}$ can be obtained at $\Lambda_{\mathrm{EW}}$. Again the results of $m_{1}, \Delta m_{31}^{2}, \theta_{12}$ and $\theta_{23}$ at $\Lambda_{\mathrm{EW}}$ are quite stable against the change of $\theta_{13}$ from $1^{\circ}$ to $5^{\circ}$ at $\Lambda_{\mathrm{SS}}$, but the result of $\Delta m_{21}^{2}$ becomes smaller.

In a similar way, one may realize the radiative generation of $\sigma=90^{\circ}$.

Finally it is worth remarking that our analysis is essentially independent of the specific textures of lepton Yukawa coupling matrices. Thus it can be applied to the concrete work of model building. Since the elegant thermal leptogenesis mechanism is usually expected to work at the seesaw scale, a study of its consequences at low-energy scales is available by means of the one-loop RGEs that we have obtained. In other words, the RGEs may serve as a useful bridge to establish a kind of connection between the phenomena of $\mathrm{CP}$ violation at low- and high-energy scales.

I apologize for not being able to cite more relevant works, just due to the page limitation.

\section{References}

[1] H. Fritzsch and Z.Z. Xing, Phys. Lett. B 517, 363 (2001); Z.Z. Xing, Int. J. Mod. Phys. A 19, 1 (2004).

[2] A. Strumia and F. Vissani, hep-ph/0503246.

[3] J.A. Casas et al., Nucl. Phys. B 573, 652 (2000). S. Antusch et al., Nucl. Phys. B 674, 401 (2003).

[4] S. Luo, J.W. Mei, and Z.Z. Xing, Phys. Rev. D 72, 053014 (2005). 